\title{
Changes in interstitial cell of Cajal-like cells density in congenital ureteropelvic junction obstruction
}

\author{
Piotr Koleda $\cdot$ Wojciech Apoznanski $\cdot$ Zdzislaw Wozniak • \\ Leslaw Rusiecki • Tomasz Szydelko • Witold Pilecki • \\ Marcin Polok · Dariusz Kalka $\cdot$ Artur Pupka
}

Received: 8 November 2010/Accepted: 12 April 2011/Published online: 6 May 2011

(C) The Author(s) 2011. This article is published with open access at Springerlink.com

\begin{abstract}
Purpose The authors examined the number of interstitial cells of Cajal-like cells (ICC-LCs) in obstructed ureteropelvic junction (UPJ) in comparison with normal UPJ specimens and age-related changes. Materials and methods A total of 20 human formalin fixed, paraffin-embedded specimens of intrinsic UPJO from children at the mean age of 8.1 years (age range: 8 months-16.8 years) and 5 control samples from children at the mean age of 2.3 years (range 2.4 months-7.4 years) were investigated immunohistochemically for the expression of
\end{abstract}

P. Koleda $(\bowtie) \cdot$ L. Rusiecki · W. Pilecki · D. Kalka Department of Pathophysiology, Wroclaw Medical University, ul. Marcinkowskiego 1, 50-368 Wroclaw, Poland

e-mail: pkoleda@koleda-pl.eu

W. Apoznanski - M. Polok

Department and Clinic of Paediatric Surgery and Urology,

Wroclaw Medical University, Wroclaw, Poland

Z. Wozniak

Department of Pathomorphology, Wroclaw Medical

University, Wroclaw, Poland

T. Szydelko

Department and Clinic of Urology, Wroclaw Medical

University, Wroclaw, Poland

A. Pupka

Department and Clinic Vascular, General and Transplantation Surgery, Wroclaw Medical

University, Wroclaw, Poland c-kit proto-oncogene by light microscopy with computerized image analysis. The results were examined by Fisher's exact test, Yates' chi-square test, and t test for Pearson's correlation coefficient. A $P$ value less than 0.05 was considered statistically significant. Results The number of ICC-LCs-dense fields was significantly higher in UPJO specimens in comparison with the normal group $(P=0.0004)$. The number of ICC-LCs-sparse fields was significantly lowered in UPJO specimens in comparison with the normal group $(P=0.0122)$. There was no significant difference in the number of ICC-LCs-medium fields in obstructed and normal UPJ specimens. The number of ICC-LCs was decreasing significantly with increasing age of the patients with UPJO $(P=0.0038)$.

Conclusions The higher density of c-kit-positive ICCLCs in UPJ may manifest the compensation of altered peristalsis in UPJO. The number of ICC-LCs-dense fields decreasing with age may show the failure of compensation and regression of the compensatory changes.

Keywords c-kit - Child - Interstitial cells of Cajallike · Obstruction · Peristalsis · Ureteropelvic junction

Ureteropelvic junction obstruction (UPJO) is regarded as the most common congenital abnormality of the ureter. According to Murnagham, the cause of the obstruction is the abnormality in the lamina muscularis of UPJ, whereas Notley and Hanna point to the 
increase in collagen between the muscle bundles and elastin in the adventitia [1-5]. Maizels and Stephens claim that UPJO results from valves produced by infoldings of ureteral mucosa $[1,6]$. On the other hand, Kiil maintains that in the fetal life, the upper ureter may be trapped behind the crossing vessels supplying the lower pole of the kidney, which hinders its development [7].

Further investigations into the etiology of UPJO supported by molecular techniques and the immunochemical analysis have shown abnormalities of ureteral innervations [8, 9]. Other studies have indicated abnormalities of microstructure as well as functional disorders impeding the urine outflow from the renal pelvis that can be regarded as intrinsic obstruction.

Recently, research has been concerned with interstitial cells of Cajal (ICCs) at the UPJ. They were described for the first time in the gastrointestinal tract by Ramon Y. Cajal in 1893. The identification of ICCs in the human urinary tract was possible due to the discovery that the tyrosine kinase receptor c-kit was expressed on the surface of the cells. This in turn made it possible to label ICCs with antibodies to the proto-oncogene c-kit [9-12]. In order to distinguish between ICCs in the urinary tract and ICCs in the gastrointestinal tract, the former are called interstitial cells of Cajal-like cells (ICC-LCs).

The expression of connexin $43(\mathrm{Cx} 43)$ indicates a potential integrating role of ICC-LCs in the propagation of pyeloureteric peristalsis. ICC-LCs are said to be involved in conducting of electrical impulses from atypical smooth muscle cells (a-SMC) to typical smooth muscle cells (t-SMC). The result is the synchronic contraction of t-SMCs propagated distally to the bladder [13, 14].

The observation that ICC-LCs express vanilloid receptor-like 1 protein (VRL-1/TRPV2) suggests the role they may have in the modulation of pyeloureteric peristalsis as sensors of physical and chemical stimuli [12].

In cases of ureteral abnormalities, ICC-LCs can take over the function of a conducting pathway, maintaining the propagation of impulses and pyelouretic peristalsis [12, 14, 15].

The objective of the following study is the analysis of changes in the density of ICC-LCs at UPJ in patients with congenital UPJO.

\section{Materials and methods}

UPJ specimens were obtained from 20 patients aged from 8 months to 16.8 years (mean age 8.1 years), who underwent pyeloplasty for intrinsic UPJO. Patients were selected for the surgery on imaging studies and renal scyntygraphy. All cases of intrinsic obstruction were confirmed intraoperatively (extrinsic obstruction was excluded from the study).

In the control group, there were 5 patients aged from 2.4 months to 7.4 years (mean age 2.3 years) who did not show any clinical or laboratory symptoms of UPJO but underwent nephrectomy because of Wilms tumor.

A UPJ sample no smaller than 5-6 mm was taken each time. For both control and patients with UPJO, UPJ was excised with the resection margin of 2-3 $\mathrm{mm}$ above and below the UPJ. Each specimen was immediately fixed in $4 \%$ formalin with phosphate buffer and embedded in paraffin.

Immunohistochemistry

For the immunodetection of proteins, 4- $\mu$ m-thick histologic sections were placed after deparaffinization in High pH Target Retrieval Solution (code S3308 Dako Cytomation, Denmark) and heated in a water bath $\left(96^{\circ} \mathrm{C}\right)$ for $20 \mathrm{~min}$ for antigen retrieval. Endogenous peroxidase activity was blocked with $3 \%$ $\mathrm{H}_{2} \mathrm{O}_{2}$. Specimens were then incubated for $30 \mathrm{~min}$ at room temperature with Polyclonal Rabbit AntiHuman CD117, c-kit (code A4502 Dako Cytomation, Denmark). Immunoreactivity was detected by using EnVision + HRP, Rabbit (code K4008, Dako Cytomation, Denmark) and visualized by 3,3-diaminobenzidine (DAB) staining. Finally, the specimens were counter-stained by immersion in hematoxylin.

\section{Light microscopy}

An optical microscope Olympus BX41 (Olympus America, Center Valley, PA, USA) with a digital camera Olympus DP/01 (Olympus America, Center Valley, PA, USA) was used in the analysis of the specimens. The end magnification was $400 \times$. The images were processed by DP Controller Ver. 1.2.1.108 (Olympus America, Center Valley, PA, USA), a computer-assisted image analyzer. 
In each specimen, 11 well-stained, oriented highpower fields of $0.136 \mathrm{~mm}^{2}$ each were evaluated, and the density of c-kit-positive ICC-LCs was graded. The grades: sparse, moderate, and many indicated $0-1,2-3$, and $4-8$ cell bodies per high-power field, respectively.

The assessment was made by two independent investigators. As two cell types showed c-kit immunoreactivity, ICC-LCs and mast cells, the former were identified in the inner border of the circular muscle layer in parallel orientation with muscle fibers. Additionally, ICC-LCs had a fusiform cell body with a thin cytoplasm with a large oval nucleus. Mast cells, on the other hand, were found in the submucosa, muscularis mucosa, and mucosa (Fig. 1). Results were examined by Fisher's exact test, Yates' chi-square test, and test for Pearson's correlation coefficient. A $P$ value less than 0.05 was considered statistically significant.

As both groups, i.e., the examined and the control one, were heterogeneous in regard to the patients' age, we tried to homogenize these groups. In the examined group, we reduced the number of cases to 7 patients with UPJO, aged 8 months- 5.2 years (the mean age 2.2 years). The control group remained unchanged and it consisted of 5 patients, aged 2.4 months -7.4 years (the mean age 2.3 years). The results obtained from the homogenous groups were statistically analyzed again with Fisher's exact test,

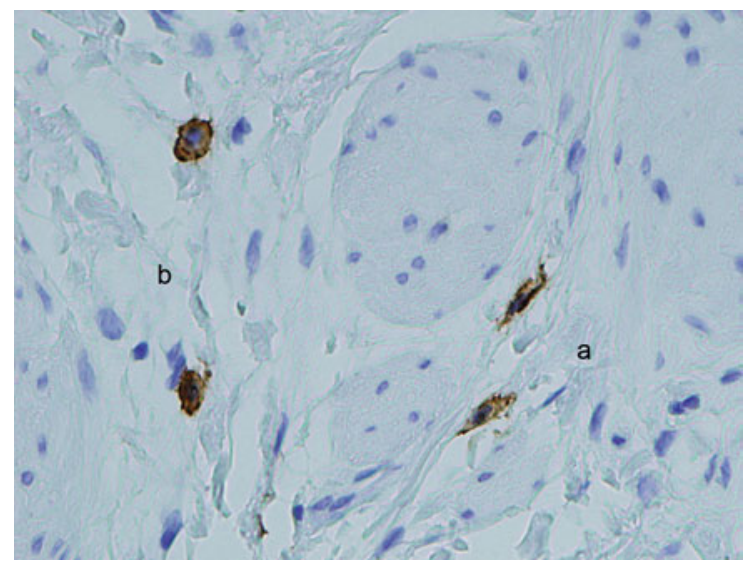

Fig. 1 C-kit-positive a interstitial cells of Cajal-like cells in the inner border of the circular muscle layer in parallel orientation with muscle fibers and $\mathbf{b}$ mast cells dispread in mucosa
Yates' chi-square test, and $t$ test for Pearson's correlation coefficient. A $P$ value less than 0.05 was considered statistically significant.

\section{Results}

The number of fields with few ICC-LCs $(0-1)$ was significantly lower in obstructed UPJ specimens than in control samples $(P=0.0122)$. The number of fields with many ICC-LCs (4-8) was significantly higher in obstructed cases than in normal UPJ $(P=0.0004)$. No statistically significant difference was found with respect to the moderate grade (Table 1, Fig. 2).

In normal UPJ, no statistically significant correlation between the density of ICC-LCs and the patient's age was observed. In obstructed UPJ specimens, the correlation was found significant: the number of ICC-LCs was decreasing with the rising age $(R=$ $-0.6167, P=0.0038$ ) (Fig. 3).

The comparison of the percentage of the fields with sparse, moderate, and many interstitial cells of Cajal-like cells in the homogenized groups yielded a result similar to that obtained in the entire examined group. In the homogenized groups, the number of fields with few ICC-LCs (0-1) was significantly lower in obstructed UPJ specimens than in control samples $(P=0.0003)$. The number of fields with many ICC-LCs (4-8) was significantly higher in obstructed cases than in normal UPJ $(P<0.0001)$. No statistically significant difference was found with respect to the moderate grade (2-3 ICC-LCs).

The analysis of the correlation between the patients' age and the number of interstitial cells of Cajal-like cells in the group of 7 patients (after homogenization) indicated that the older the patient, the lower the number of ICC-LCs. The correlation was found statistically significant $(P=0.0416$, $R=-0.7730)$.

\section{Discussion}

Increased expression of c-kit-positive ICC-LCs in congenital UPJO may indicate the development of a compensatory mechanism for the failure of urine to be propelled from the renal pelvis through the ureter. This surprising result entails the following 
Table 1 The comparison of the number and percentage of the fields with sparse, moderate, and many interstitial cells of Cajal-like cells in patients with congenital ureteropelvic junction obstruction and control group

\begin{tabular}{lllll}
\hline & Total fields no. & $\begin{array}{l}\text { ICC-LCs-sparse (0-1) } \\
\text { fields no. }\end{array}$ & $\begin{array}{l}\text { ICC-LCs-moderate (2-3) } \\
\text { fields no. }\end{array}$ & $\begin{array}{l}\text { ICC-LCs-many (4-8) } \\
\text { fields no. }\end{array}$ \\
\hline UPJO group & $220(100 \%)$ & $56(25.5 \%)$ & $100(45.5 \%)$ & $64(29 \%)$ \\
Control group & $55(100 \%)$ & $24(43.6 \%)$ & $27(49.1 \%)$ & $4(7.3 \%)$ \\
Difference $(P)$ & & $<0.05$ & NS & $<0.05$ \\
\hline
\end{tabular}

no. number, ICC-LCs interstitial cells of Cajal-like cells, $N S$ not statistically significant

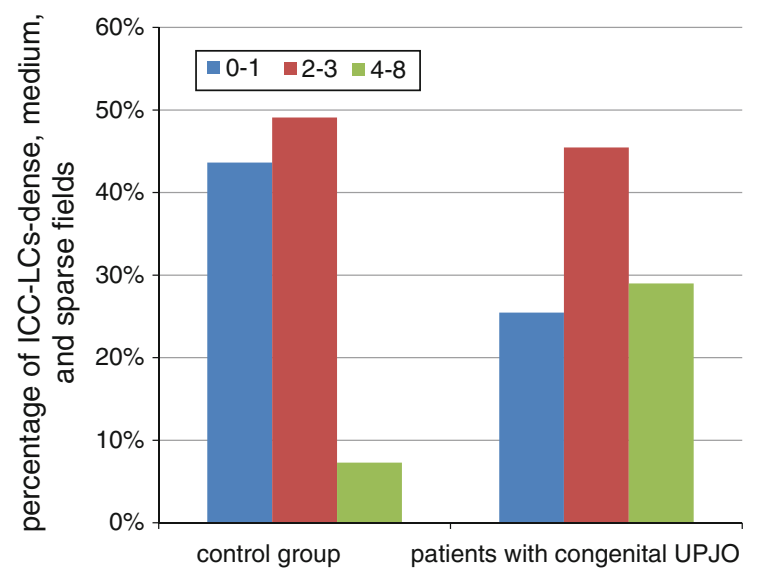

Fig. 2 The comparison of the percentage of the fields with sparse, moderate, and many interstitial cells of Cajal-like cells in patients with congenital ureteropelvic junction obstruction and control group

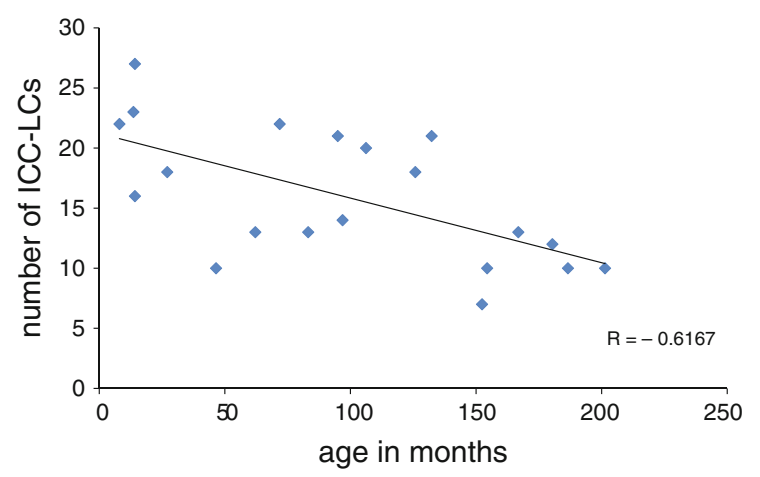

Fig. 3 The correlation between age of the patients with congenital ureteropelvic junction obstruction and number of interstitial cells of Cajal-like cells

hypothesis: If the lower density of c-kit-positive ICC-LCs in UPJ is not the cause of UPJO, then the abnormality may result from the abnormal distribution of c-kit-positive ICC-LCs in patients with congenital UPJO. However, this abnormal distribution was not found in our patients with congenital UPJO. In the obstructed UPJ specimens with a large number of c-kit-positive ICC-LCs, the fields with no c-kit-positive ICC-LCs were statistically rarer.

In 1992, Starr indicated muscular hypertrophy and the markedly increased number of UPJ longitudinal muscle bundles in infants with UPJO as compared to healthy babies [16]. In 1996, Seremetis and Maizels reported increased transforming growth factor- $\beta$ (TGF- $\beta$ ) expression in the renal pelvis in patients with UPJO [1, 9, 17, 18].

Ekinci et al. indicated increased amplitude and frequency of spontaneous renal pelvic contractility as a response to UPJO. The above increase may be, according to the study, a consequence of overactivity (hyperactivity) and growth of pace-making cells [19].

Bearing in mind the role of ICC-LCs in the propagation of electrical events and in the modulation of neurotransmission, the increase in their number and activity as a consequence of UPJ muscular hypertrophy seems natural.

Our observations are in disparity with Solari et al.'s and Yang et al.'s findings, who indicated the decreased density of c-kit-positive ICC-LCs in children with UPJO $[10,20]$. It seemed to us at first that the differences in the results could be attributed to the differences in the mean age of the examined patients. However, the results we obtained for the homogenized group involving 7 patients aged 2.2 years on average, the mean age being similar to that in Solari et al. (2.3 years), were the same as the ones observed for the entire group. Furthermore, the correlation between the patients' age and the number of interstitial cells of Cajal-like cells was more conspicuous in the 7-patient group than it was in the large group of 20 patients (fewer cells were observed in older patients). Yang et al. do not provide the value of the 
patients mean age; therefore, we could not compare our results with theirs.

Perhaps the differences between our results and those reported by Solari et al. and Yang et al. can be explained referring to the observations on animal data. Chang et al. as well as Der et al. (both quoted by Yang et al.) indicated considerable plasticity of Cajal cells in the temporary obstructed small intestine or inflammation-induced motor dysfunction of the small bowel. Their observations suggest that bowel obstruction results in changes in c-kit expression on ICCs rather than in changes of their density [20-22]. It is not unlikely that similar processes take place in the urinary tract. If so, then the differences between our results and those obtained by Solari et al. and Yang et al. may be connected with the change in c-kit expression on ICCs resulting from a different degree or different duration of an obstruction rather than with the density of ICCs.

Kuzgunbay et al.'s observations regarding the effects of acquired ureteral obstruction on the number and morphology of ICC-LCs in rats are noteworthy, too. A sudden increase in ICC-LCs to the peak level was detected in the second week following the acquisition of an obstruction. Then the number of c-kit-positive ICC-LCs gradually decreased until it reached plateau after 1-2 months [23]. The results obtained in the following study seem to corroborate the above observations. The density of c-kit-positive ICC-LCs was markedly increased in patients with UPJO as compared to the control group. The number of c-kit-positive ICC-LCs was found to be decreasing with the growing age of patients, that is with the obstruction duration time. As in the control group, no correlation was found between the number of c-kitpositive ICC-LCs and the patients' age, it seems that the only explanation might be gradual regression of the changes compensating for the obstruction in UPJ.

We do believe that Wilms tumor is the main, if not the only, affliction that makes it possible to obtain the unaffected UPJ for the analysis in the control group. The limitation of our study is a low number of cases in the control group because very few patients with Wilms tumor are treated yearly in our institution. Solari et al. must have faced similar problems as their control group consisted of 7 patients [10].

Open Access This article is distributed under the terms of the Creative Commons Attribution Noncommercial License which permits any noncommercial use, distribution, and reproduction in any medium, provided the original author(s) and source are credited.

\section{References}

1. Carr MC, El-Ghoneimi A (2007) Anomalies and surgery of the ureteropelvic junction. In: Campbell-Walsh urology, Section XVII: pediatric urology, 9th edn. Saunders Elsevier, pp 3359-3382

2. Murnaghan GF (1958) The physiology of hydronephrosis. Postgrad Med J 34(389):143-148

3. Notley RG (1968) Electron microscopy of the upper ureter and the pelvi-ureteric junction. Br J Urol 40(1):37-52

4. Hanna MK, Jeffs RD, Sturgess JH et al (1976) Ureteral structure and ultrastructure. Part I: the normal human ureter. Urol 116:718-724

5. Hanna MK, Jeffs RD, Sturgess JH et al (1976) Ureteral structure and ultrastructure: part 11: congenital hydronephrosis and primary obstructive megaureter. J Urol 116:725-729

6. Maizels M, Stephens FD (1980) Valves of the ureter as a cause of primary obstruction of the ureter: anatomic, embryologic and clinical aspects. J Urol 123:742-747

7. Kiil F (2002) Analysis of myogenic mechanisms in renal autoregulation. Acta Physiol Scand 174(4):347-355

8. Krakos M, Andrzejewska E (2004) Abnormalities of ureteral innervation as the base of etiopathogenesis of congenital hydronephrosis in children. Przegl Pediatr 34(2):94-97

9. Wang Y, Puri P, Hassan J et al (1995) Abnormal innervations and altered nerve growth factor messenger ribonucleic acid expression in ureteropelvic junction obstruction. J Urol 154:679-683

10. Solari V, Piaseczna-Piotrowska A, Puri P (2003) Altered expression of interstitial cells of Cajal in congenital ureteropelvic junction obstruction. J Urol 170:2420-2422

11. Metzger R, Schuster T, Till H et al (2005) Cajal-like cells in the upper urinary tract: comparative study in various species. Pediatr Surg Int 21:169-174

12. Metzger R, Schuster T, Till H et al (2004) Cajal-like cells in the human upper urinary tract. J Urol 172(2):769-772

13. van der Aa F, Roskams T, Blyweert W et al (2004) Identification of kit positive cells in the human urinary tract. J Urol 171(6 Pt1):2492-2496

14. Lang RJ, Tonta MA, Zoltkowski BZ et al (2006) Pyeloureteric peristalsis: role of atypical smooth muscle cells and interstitial cells of Cajal-like cells as pacemakers. J Physiol 576(Pt3):695-705

15. Lang RJ, Klemm MF (2005) Interstitial cell of Cajal-like cells in the upper urinary tract. $\mathrm{J}$ Cell Mol Med 9(3):543-556

16. Starr NT, Maizels M, Chou P et al (1992) Microanatomy and morphometry of the hydronephrotic "obstructed" renal pelvis in asymptomatic infants. J Urol 148(2 Pt2):519-524

17. Palmer LS, Maizels M, Kaplan WE et al (1997) Urine levels of transforming growth factor-beta 1 in children with ureteropelvic junction obstruction. Urol 50(5):769-773 
18. Seremetis GM, Maizels M (1996) TGF-beta mRNA expression in the renal pelvis after experimental and clinical ureteropelvic junction obstruction. $\mathrm{J}$ Urol 156(1):261-266

19. Ekinci S, Ertunc M, Ciftci AO et al (2004) Evaluation of Pelvic contractility in ureteropelvic junction obstruction: an experimental study. Eur J Pediatr Surg 14:93-99

20. Yang X, Zhang Y, Hu J (2009) The expression of Cajal cells at the obstruction site of congenital pelviureteric junction obstruction and quantitative image analysis. J Pediatr Surg 44:2339-2342
21. Chang IY, Glasgow NJ, Takayama I et al (2001) Loss of interstitial cells of Cajal and development of electrical dysfunction in murine small bowel obstruction. J Physiol 536:555-568

22. Der T, Bercik P, Donnelly G et al (2000) Interstitial cells of Cajal and inflammation-induced motor dysfunction in the mouse small intestine. Gastroenterology 119:1590-1599

23. Kuzgunbay B, Doran F, Bayazit Y et al (2009) The effects of ureteral obstruction on Cajal-like cells in rats. J Pediatr Urol 5(4):269-273 